\title{
The fastscanning observing technique for millimeter and submillimeter astronomy
}

\author{
L. A. Reichertz, B. Weferling, W. Esch, and E. Kreysa \\ Max-Planck-Institut für Radioastronomie (MPIfR), Auf dem Hügel 69, 53121 Bonn, Germany \\ Received 22 June 2001 / Accepted 30 August 2001

\begin{abstract}
Fastscanning is a new observing technique for millimeter and submillimeter astronomy from ground based telescopes. The atmosphere is eliminated by taking advantage of detector arrays. Instead of wobbling the secondary mirror with a fixed frequency of a few $\mathrm{Hz}$ to filter the atmospheric contribution, we sample the detector outputs at a much higher rate without a modulation by the secondary mirror. The atmospheric contribution is then removed later in the offline data reduction by correlation analysis between the detector pixels. In order to satisfy the AC requirement of the amplifiers in the absence of modulation, the telescope scans fast to convert the spatial frequencies of the sky into the detector frequency band. The acquired AC signals are then deconvolved with the corresponding filter function in order to reconstruct quasi-DC signals. This article describes the technique of this new method and shows simulations and preliminary test results.
\end{abstract}

Key words. methods: observational - techniques: photometric - atmospheric effects

\section{Introduction}

Astronomical observations from ground based telescopes in the (sub)millimeter wavelength regime are strongly compromised by fluctuations of the atmospheric emission. This is especially true for broadband measurements with sensitive detectors like bolometers. In order to subtract the atmospheric contribution from the measurement, dual beam techniques have been used for many years. A common method is to use a chopping (wobbling) secondary mirror which alternatively points the beam to two adjacent positions on the sky. After phase sensitive detection, spatial and temporal emission fluctuations should cancel, at least to first order. Since the introduction of the EKH algorithm (Emerson et al. 1979) this technique is applied even to extended sources which are many times larger in angular extent than the separation of the beams. Invented originally for single pixel detectors, this method is used today even with large arrays of detectors.

Despite the great success of this technique, a wobbling secondary mirror has some major disadvantages: A fast, precisely moving mirror is quite a technical challenge, particularly for large telescopes. Therefore, actually only low modulation frequencies (e.g. $2 \mathrm{~Hz}$ at the $30 \mathrm{~m}$ Millimeter Radio Telescope (MRT)) are possible. This limits the scan velocity in mapping modes and makes it difficult to achieve the required noise limit in readout electronics, as

Send offprint requests to: L. A. Reichertz, e-mail: reichertz@mpifr-bonn.mpg.de will be described in Sect. 2. The mechanics of a wobbling mirror usually allows only a certain direction of the movement which restricts the coordinate system of the observing modes. It is also a source of vibrations that can lead to microphonics in the signals of highly sensitive bolometers. Small asymmetries in the wobbling can lead to large offsets, due to different optical paths for each beam. Last but not least, a wobbling secondary mirror is not available on every telescope where continuum observations are of interest. In this paper we present a new mapping technique where a wobbling secondary mirror is no longer necessary.

\section{The fastscanning principle}

In contrast to the chopping secondary method which samples the atmospheric emission in the two beams with a relative shift of half a period, this new method takes advantage of the fact that in detector arrays all beams are receiving atmospheric emission simultaneously. The atmospheric contribution is then removed during the data reduction by correlation analysis between the detector pixels.

The realisation of this quite simple idea has non-trivial consequences for the data acquisition principles and readout electronics. This will be explained in the following. Along with the creation of a dual beam, the wobbling mirror has a second function: The signal of interest is modulated at the wobbling frequency and this allows the application of a phase-sensitive demodulation (Lock-In) technique. Readout electronics for bolometers are therefore 


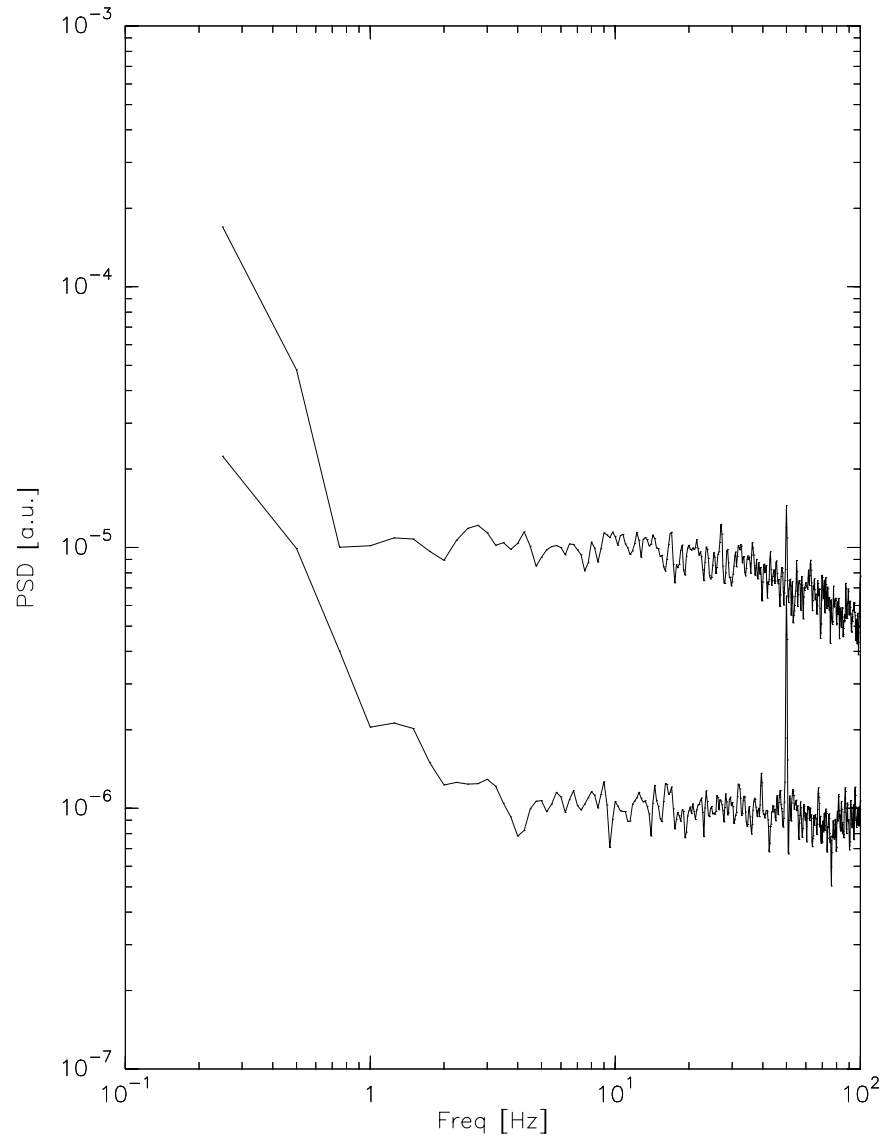

Fig. 1. Noise spectra of bolometer signal (upper curve) and amplifier (lower curve).

optimized for low frequency AC signals. Figure 1 shows a typical noise spectrum of a Ge-bolometer of the MPIfR array MAMBO (Kreysa et al. 1999). The amplifier for the bolometer signals should not contribute significantly to the noise equivalent power of the bolometer. With DC bias this condition can be met only above a low frequency limit below which the noise rises sharply due to thermal drifts and electronic $1 / f$ noise. With selected transistors, this $1 / f$ knee can be pushed down to about $1 \mathrm{~Hz}$, just below the frequencies of common wobblers. The steep rise of the noise towards zero frequency makes it absolutely necessary to use $\mathrm{AC}$ coupling, which is actually a $R C$ high pass filter at the input of the amplifier. The complex frequency function of this filter is given by the equation

$A(\nu)=\frac{1}{1+1 /(i 2 \pi \nu R C)}$.

We chose resistor $R$ and capacitor $C$ for a time constant of $\tau_{\mathrm{amp}}=R C=1.32 \mathrm{~s}$, corresponding to a $3 \mathrm{~dB}$ rolloff at $0.12 \mathrm{~Hz}$. The idea of fastscanning is now to generate $\mathrm{AC}$ signals that can pass this filter without using a modulation by a wobbler. Therefore we let the telescope scan relatively fast to convert the spatial frequencies of a structure on the sky into signal frequencies inside the filter pass band. Applying AC bias would shift the $1 / f$ knee to lower frequencies and would therefore allow slower scanning at the cost of higher electronic complexity. We chose

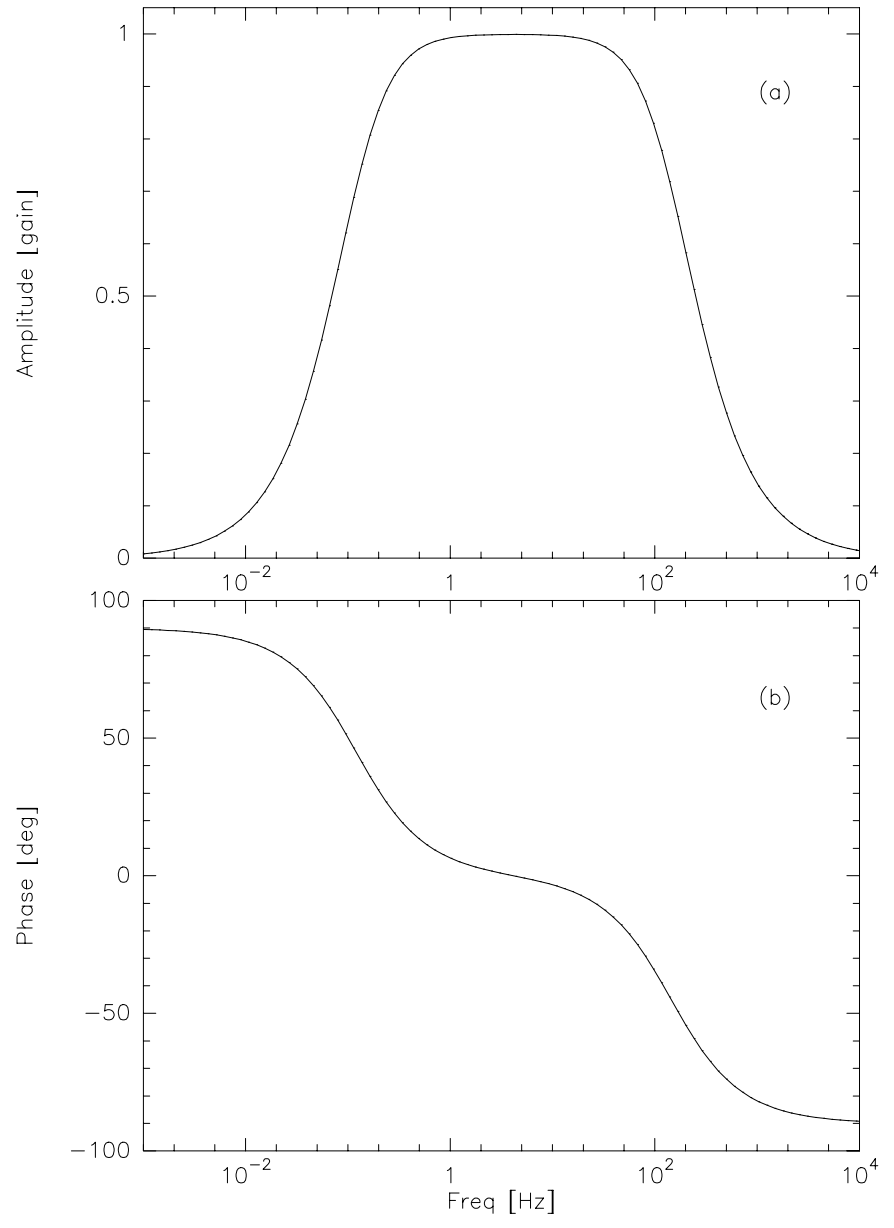

Fig. 2. a) Amplitude and b) phase of the complex bolometer filter function.

electronic simplicity and fastscanning. With respect to sky emission fluctuations, there might be intrinsic advantages in fastscanning. This will be the subject of further investigations.

\section{The bolometer filter function}

The responsivity of a bolometer is a function of its heat capacity $C_{\mathrm{H}}$ and the thermal conductance $G_{\mathrm{T}}$ of the thermal link. Although these are rather complicated functions of temperature and other parmeters, in a first order approximation for small signals they can be regarded as constant and the bolometer can be described in a simple model with an effective thermal time constant (e.g. Richards 1994)

$\tau_{\text {bol }}=\frac{C_{\mathrm{H}}}{G_{\mathrm{T}}}$.

In this model the frequency dependence of the bolometer resonse $S(\nu)$ then follows the equation of a low pass filter

$S(\nu)=S(0) \frac{1}{1+i 2 \pi \nu \tau_{\mathrm{bol}}}$.

For the bolometers of MAMBO we determined a time constant of $\tau_{\text {bol }}=1.1 \mathrm{~ms}$ by measuring the amplitude and the phase of the bolometer response to the sinusodial chopped 


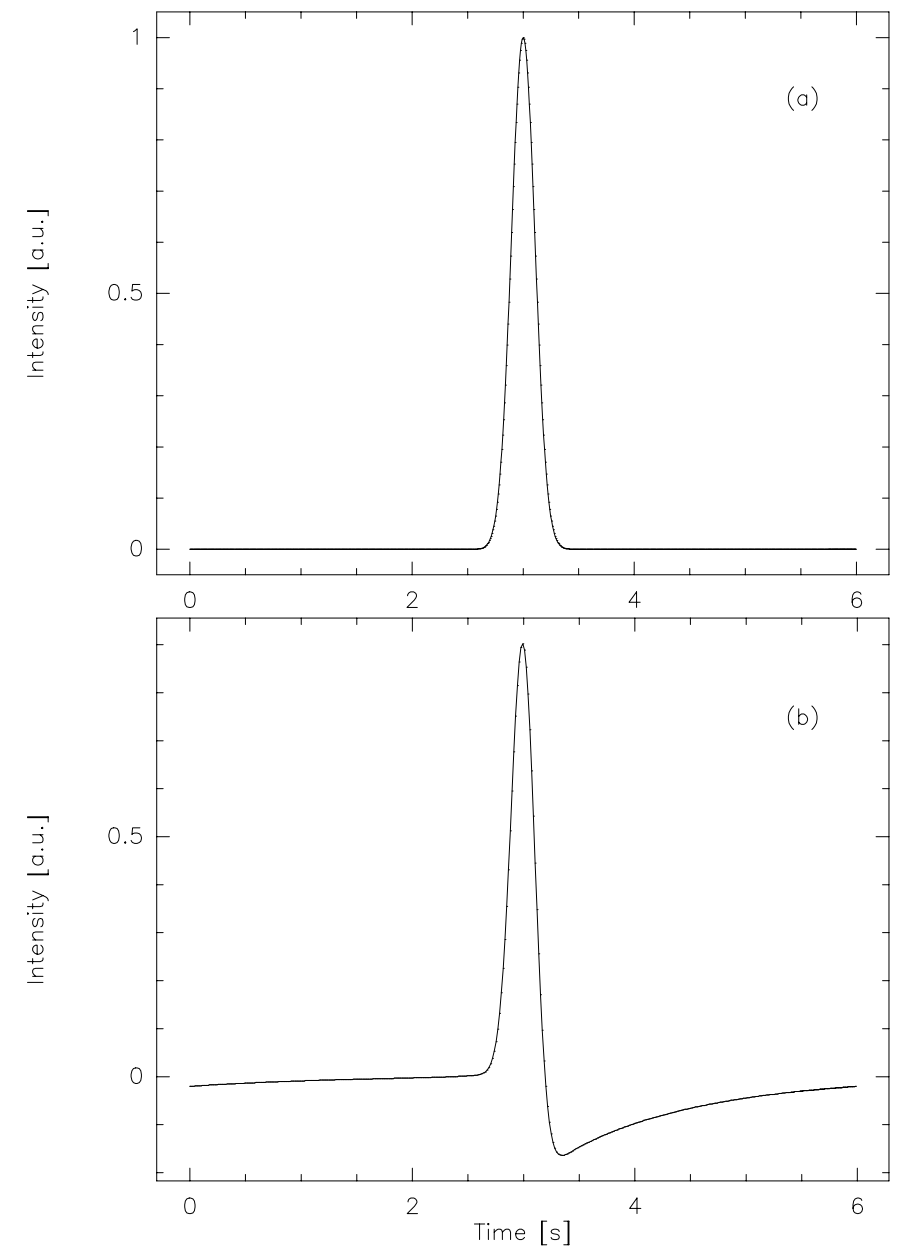

Fig. 3. Simulated point source scan: a) before and b) after convolution with the filter function.

radiation of a black body source at different chopper frequencies. Combining the high pass associated AC coupling (Eq. (1)) with the bolometer response function (Eq. (3)) yields a certain frequency passband

$G(\nu)=\frac{1}{\left(1+1 /\left(i 2 \pi \nu \tau_{\mathrm{amp}}\right)\right)\left(1+i 2 \pi \nu \tau_{\mathrm{bol}}\right)}$.

The detector system is sensitive only to power variations with frequencies inside this band. We call this the bolometer filter function. This complex function is shown with its amplitude and phase in Fig. 2. Any signal that is applied to the detector system during the scanning process is convolved with this bolometer function. This is shown in the following section.

\section{Simulations}

In order to study the expected signals, computer simulations were performed with several parameter sets. Figure 3 shows an example with realistic assumptions. In this simulation a $30 \mathrm{~m}$ telescope scan across a point source was assumed. At $250 \mathrm{GHz}$, the beam has a beamwidth of about 10.5 arcsec FWHM. With a scanning velocity of $40 \mathrm{arcsec} / \mathrm{s}$, a scan length of $240 \mathrm{arcsec}$ and the assumption of a Gaussian beam, this results in an input signal for

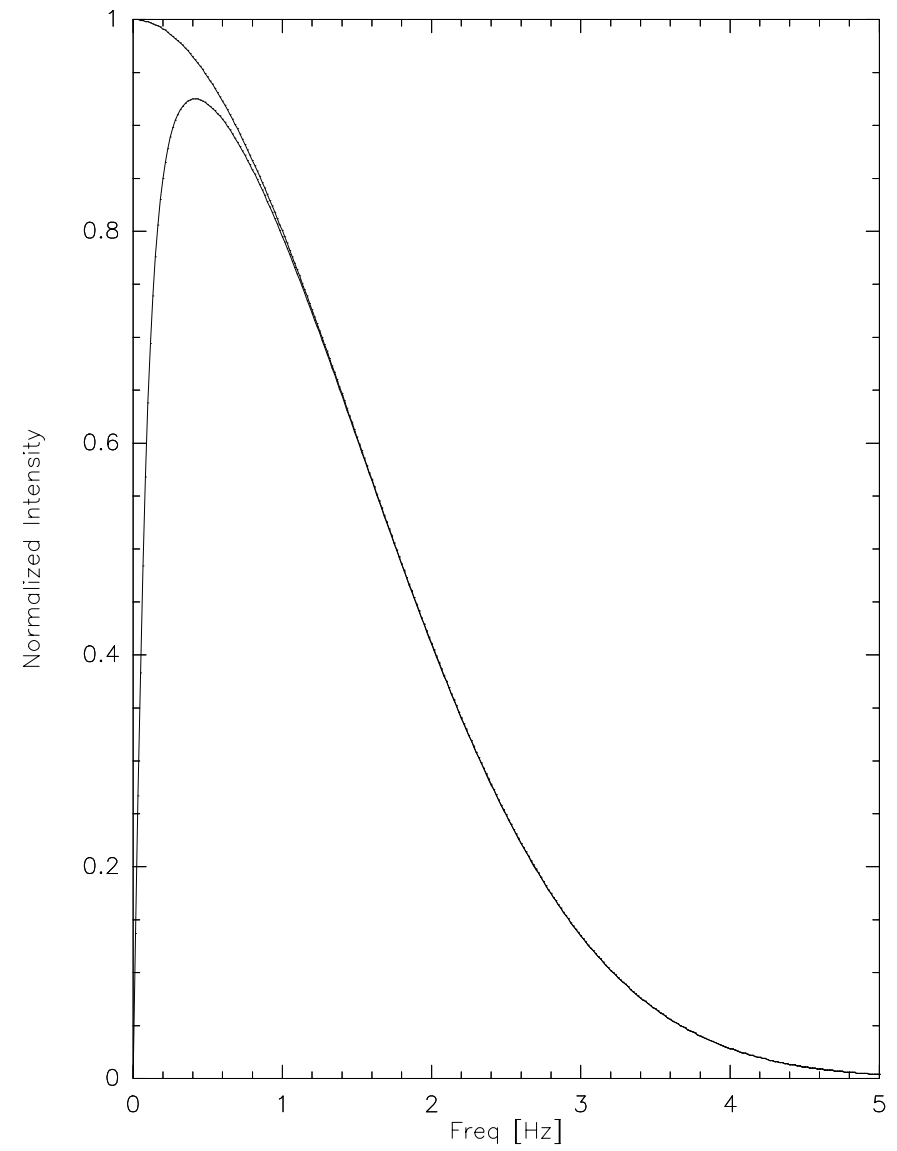

Fig. 4. Point source scan in frequency space: before (upper curve) and after (lower curve) multiplication with the filter function.

the bolometers as shown in Fig. 3a. The convolution of this signal with the bolometer filter function is illustrated in the following. Figure 4 shows the frequency spectrum of the original signal and after multiplication with Eq. (4). The resulting signal after the transformation back to the time space is displayed in Fig. 3b. This is the signal we expect to acquire in the absence of noise. To get back to the original Gaussian shape of the beam on a point source, the measured data need to be deconvolved by the inverse procedure.

Figure 4 indicates that even for high scanning velocities and the smallest possible structure, namely a point source, only relatively low signal frequencies are generated. That means, for bolometers with a time constant like the ones in MAMBO, the effect of the bolometer response is negligible in the analysis of the convolution. Furthermore, it shows the signal losses which occur due to the attenuation of the lowest frequencies. The ratio of the integral values for both spectra is a measure of the efficiency and can be calculated as a function of the three parameters scanning velocity $v$, beamwidth $d$ and source extension $s$. We begin with the input signal in Fig. 3a

$f(t)=\mathrm{e}^{-\frac{t^{2}}{2 \sigma^{2}}}$ 


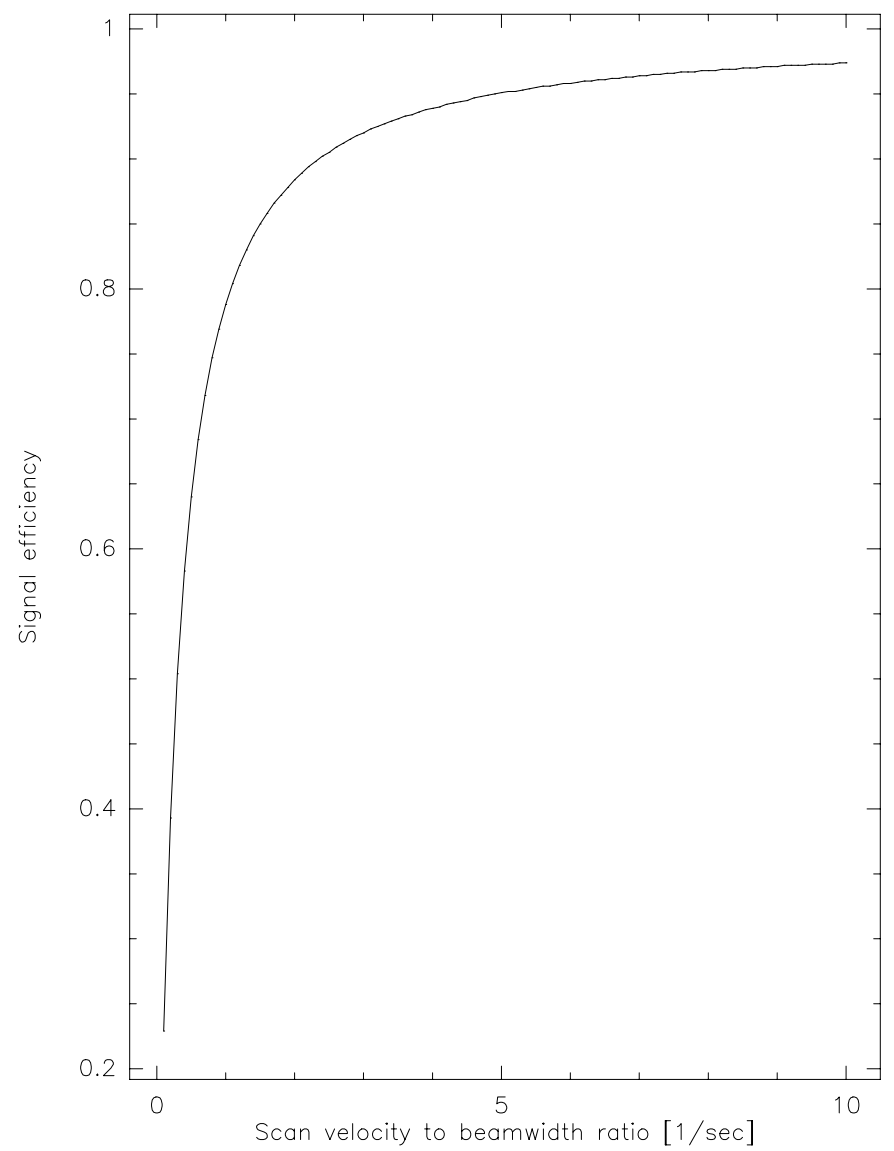

Fig. 5. Signal efficiency as a function of the scan velocity to beamwidth ratio for a scan on a point source.

with

$\sigma=\frac{d / v}{2 \sqrt{\ln 4}}$

The Fourier transform of Eq. (5) is

$F(\nu)=\sigma \mathrm{e}^{-8 \sigma^{2} \nu^{2}}$.

For an extended source this equation has to be multiplied by the Fourier transform $B(\nu)$ of its brightness distribution function, e.g. for a planet this would be the Fourier transform of the heavyside function, which is

$B(\nu)=\sqrt{\frac{2}{\pi}} \frac{\sin (s \nu / 2)}{\nu}$.

Hence, the formula for the efficiency is given by

$\eta=\frac{\int_{0}^{\infty} F(\nu) B(\nu) \frac{1}{\sqrt{1+1 / 2 \pi \nu \tau_{\mathrm{amp}}}} \mathrm{d} \nu}{\int_{0}^{\infty} F(\nu) B(\nu) \mathrm{d} \nu}$.

As an example, the efficiency is plotted in Fig. 5 for a point source $(s=0)$ as a function of the scan velocity to beamwidth ratio $v / d$.

\section{Experimental setup and first test results}

The following experimental setup was used to test the method at the $30 \mathrm{~m}$ MRT with real data. A fast readout

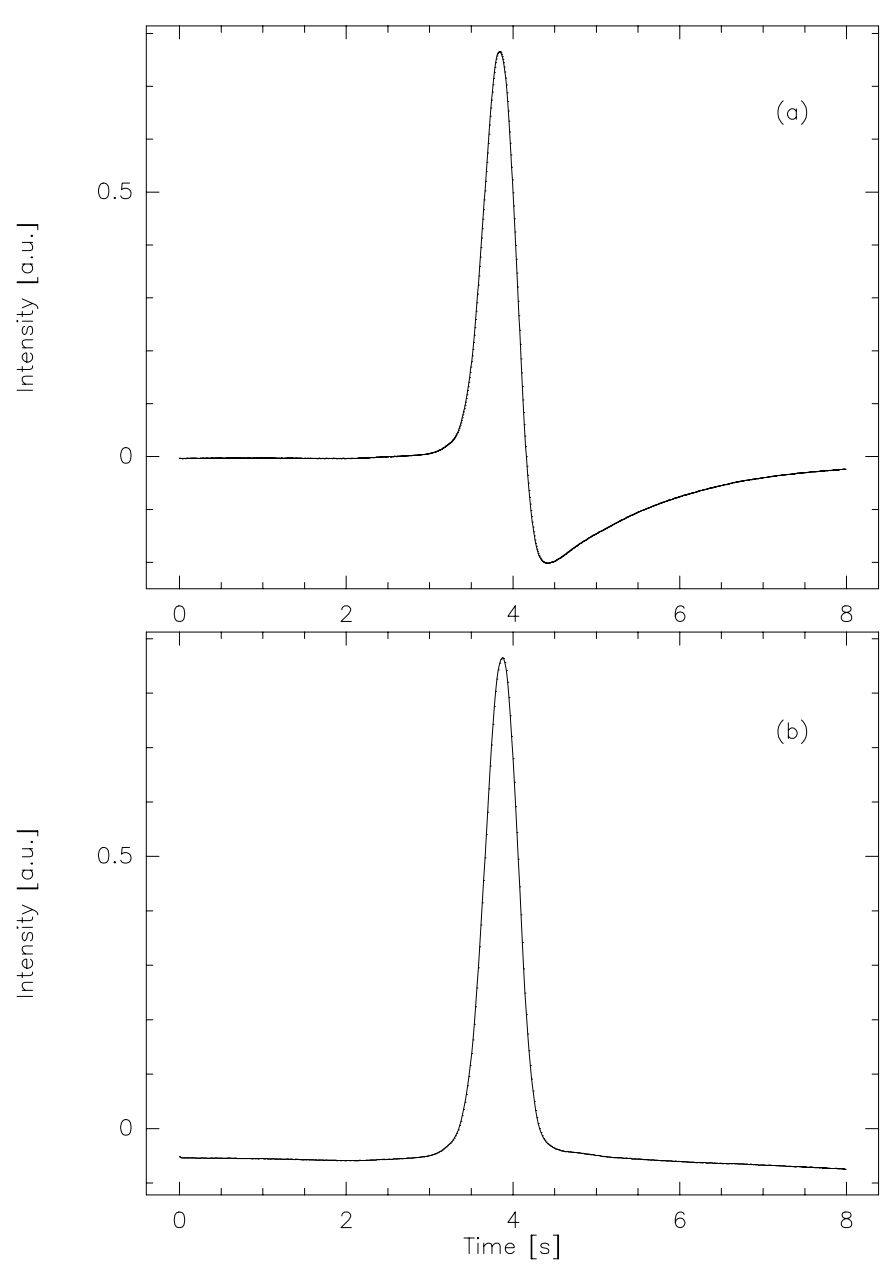

Fig. 6. Experimental result of a scan on Saturn: a) raw signal of the center channel b) after the deconvolution.

of the 37 channels of the MAMBO array was necessary. We chose a commercial off-the-shelf 16 bit A/D converter module with 64 multiplexed input channels that was plugged into the PCI bus of a PC. To avoid excess noise due to aliasing errors, we included anti-aliasing filters between the bolometer amplifier and the A/D converter. These filters modified the bolometer filter function in such a way that the roll-off for higher frequencies became steeper. After inclusion of the filters it was checked that the bolometer noise was well sampled at the frequency of $125 \mathrm{~Hz}$. During the observations the data of each bolometer channel, sampled at a rate of $125 \mathrm{~Hz}$, were streamed to a local harddisk of the PC together with an absolute timestamp.

Figure 6 shows the result of a scan on Saturn obtained at the $30 \mathrm{~m}$ telescope. The scan duration was $8 \mathrm{~s}$ with a scan velocity of $40 \mathrm{arcsec} / \mathrm{s}$. Saturn had a $250 \mathrm{GHz}$ flux of $635 \mathrm{Jy}$ at that time. In this figure the signal of the central array channel is shown. Figure 6a shows the raw signal without deconvolving the data by the bolometer filter function and Fig. $6 \mathrm{~b}$ shows the result after the raw data were deconvolved. One can see that the distortion of the signal due to the filter function is removed, the original 


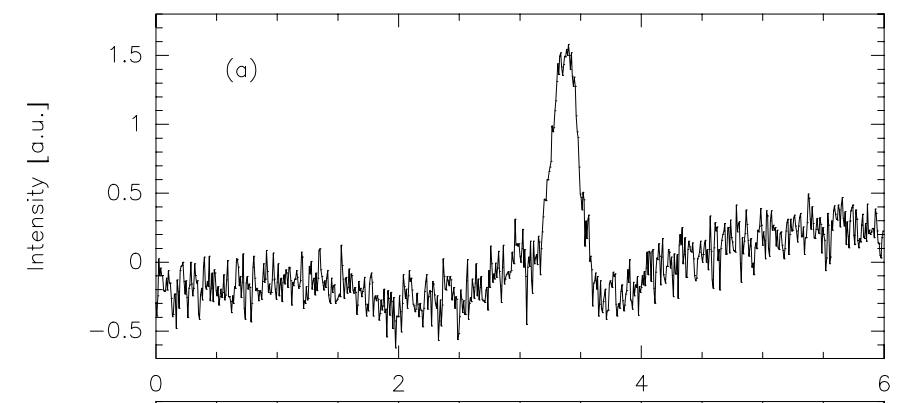

(b)

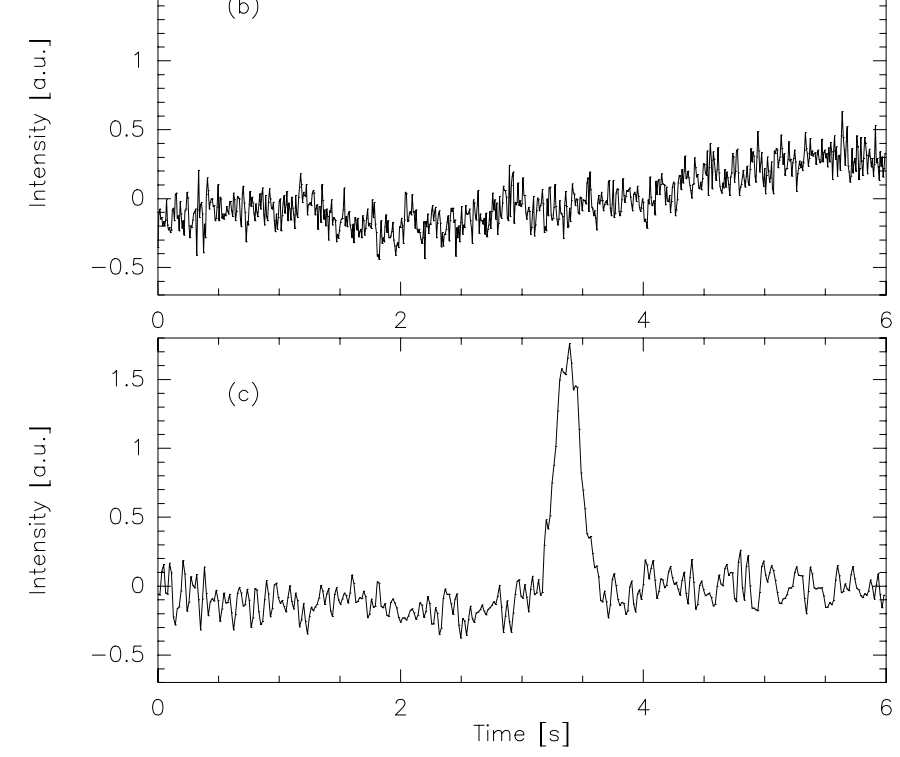

Fig. 7. Experimental result of a scan on a 7 Jy point source: signal of the center array channel after removing the atmospheric noise by substracting the signal of another channel, a) raw signal of the center channel b) array channel without source, c) difference including the deconvolution.

signal from the telescope beam on a point-like source is obtained as expected from the simulations.

In the next example the measurement of the source K3-50A with $250 \mathrm{GHz}$ flux of $7 \mathrm{Jy}$ is presented. This weaker source shows the effect of the atmospheric noise as a variable baseline (Fig. 7a). A neighbouring channel of the array in Fig. $7 \mathrm{~b}$ that did not scan the source at the same time has the same structure in the baseline and demonstrates the strong correlation of the sky emission in different channels. This signal can be used to remove the atmospheric contribution in Fig. 7a. The result is shown in Fig. 7c. Restoring the original signal by the deconvolving procedure allows Fourier filtering of noise frequencies which are higher than the signal frequencies at the same time. Under the weather conditions of this preliminary test example the resulting rms noise is reduced by about a factor of 2 . Due to the fast and simultaneous sampling in the fastscanning technique in combination with the strong correlation of the atmospheric signal in neighbouring bolometer channels, fastscanning seems to be a very promising method to remove atmospheric noise more efficiently than in the common way by using a wobbling mirror. A more detailed and quantified study of observations with the fastscanning method is currently being performed by our group and is subject of another article (Weferling et al. 2001). This includes more telescope tests on different sources and under different atmospheric conditions. It will present the data reduction for fastscanning maps and sophisticated skynoise reduction algorithms.

\section{Conclusions}

1. With arrays of bolometers it is possible to perform millimeter observations of weak sources without using a wobbling secondary mirror.

2. A fastscanning telescope is necessary to generate AC signals with a sufficient frequency bandwidth.

3. Determining the bolometer filter function and deconvolving the data with this function allows the reconstruction of the original photometric signal.

Acknowledgements. The authors would like to thank D. Muders, G. Siringo, and the IRAM staff for their support during the tests at the telescope.

\section{References}

Emerson, D. T., Klein, U., \& Haslam, C. G. T. 1979, A\&A, 76,92

Kreysa, E., Gemünd, H.-P., Gromke, J., et al. 1999, Infrared Phys. Techn., 40, 191

Richards, P. L. 1994, J. Appl. Phys., 76(1), 1

Weferling, B., Reichertz, L. A., Schmid-Burgk, J., \& Kreysa, E. 2001, A\&A, accepted 